\title{
A Trisilyl Zincate Containing Bidentate $\left[\left(\mathrm{Me}_{3} \mathrm{Si}\right)_{2} \mathrm{Si}-\left(\mathrm{CH}_{2}\right)_{2}-\mathrm{Si}\left(\mathrm{SiMe}_{3}\right)_{2}\right]^{2-}$
}

\section{Ligands}

Jaime B. Woods, Xianghua Yu, Tianniu Chen and Zi-Ling Xue*

Department of Chemistry, The University of Tennessee, Knoxville, Tennessee 37996

\section{Supporting Information}




\section{Experimental Section}

General Procedures. All manipulations were performed under a dry nitrogen or argon atmosphere with the use of either a glovebox or standard Schlenk techniques. NMR solvents were dried and stored over $5 \AA$ molecular sieves. $\mathrm{ZnCl}_{2}$ (Mallinckrodt, Analytical reagent) was purified by refluxing in $\mathrm{SOCl}_{2}$. $\mathrm{SOCl}_{2}$ was then removed under vacuum at $10^{-3}$ torr. $[\mathrm{K}(18-$ crown-6) $]_{2}\left[\left(\mathrm{Me}_{3} \mathrm{Si}\right)_{2} \mathrm{Si}-\left(\mathrm{CH}_{2}\right)_{2}-\mathrm{Si}\left(\mathrm{SiMe}_{3}\right)_{2}\right]$ was prepared by the literature method. ${ }^{9}$ Dimethoxyethane (Fisher, Certified), toluene (Fisher, Certified ACS), pentane (Fisher, Reagent grade) and $\mathrm{Et}_{2} \mathrm{O}$ (Fisher, Certified ACS) were purified by distillation from potassium benzophenone ketyl. A glass-coated stir bar was used in reactions. NMR spectra were recorded on either a Bruker AC-250 or an AMX-400 Fourier transform spectrometer. ${ }^{1} \mathrm{H}$ and ${ }^{13} \mathrm{C}$ NMR spectra were referenced to solvents (residual protons in the ${ }^{1} \mathrm{H}$ spectra). ${ }^{29} \mathrm{Si}$ chemical shifts were referenced to $\mathrm{SiMe}_{4}$. Elemental analyses were carried out by Complete Analysis Laboratory, Inc., Parsippany, New Jersey.

For preparation of the $\mathrm{Zn}$ Complex $\left[\mathrm{K}(18 \text {-crown-6) }]_{2}\left\{\left[\eta^{2}\left(\mathrm{Me}{ }_{3} \mathrm{Si}\right)_{2} \mathrm{Si}-\left(\mathrm{CH}_{2}\right)_{2}-\right.\right.\right.$ $\left.\left.\mathrm{Si}\left(\mathrm{SiMe}_{3}\right)_{2}\right]_{2} \mathrm{Zn}_{2}\left[\mu-\left(\mathrm{Me}_{3} \mathrm{Si}\right)_{2} \mathrm{Si}-\left(\mathrm{CH}_{2}\right)_{2}-\mathrm{Si}\left(\mathrm{SiMe}_{3}\right)_{2}\right]\right\}$ (1), See ref./footnote 12. ${ }^{1} \mathrm{H}$ NMR (250.1 MHz, THF- $\left.d_{8}, 23{ }^{\circ} \mathrm{C}\right) \delta 3.61$ (s, $\left.48 \mathrm{H}, \mathrm{O}-\mathrm{CH}_{2}\right), 1.26$ (s, 4H, $\mathrm{CH}_{2}$-bridging), 1.13 (s, 8H, $\mathrm{CH}_{2^{-}}$ chelating), 0.18 (s, 36H, SiMe 3 -bridging), 0.03 (s, 72H, SiMe 3 -chelating). ${ }^{13} \mathrm{C}\left\{{ }^{1} \mathrm{H}\right\}$ NMR (62.9 MHz, THF- $\left.d_{8}, 23{ }^{\circ} \mathrm{C}\right) \delta 71.27\left(\mathrm{O}-\mathrm{CH}_{2}\right), 14.63\left(\mathrm{CH}_{2}\right.$-bridging $), 12.17\left(\mathrm{CH}_{2}\right.$-chelating $), 4.52$ ( $\mathrm{SiMe}_{3}$-bridging), 3.77 ( $\mathrm{SiMe}_{3}$-chelating). ${ }^{29} \mathrm{Si}\left\{{ }^{1} \mathrm{H}\right\} \mathrm{NMR}\left(79.49 \mathrm{MHz}, \mathrm{THF}-d_{8}, 23{ }^{\circ} \mathrm{C}\right) \delta-9.95$ ( $\mathrm{SiMe}_{3}$-chelating), -11.15 ( $\mathrm{SiMe}_{3}$-bridging), -85.15 (Si-SiMe -bridging), -87.27 ( $\mathrm{Si}^{-\mathrm{SiMe}_{3}-}$ chelating).

X-ray Crystal Structure Determination of 1. The crystal structure of $\mathbf{1}$ was determined on a Bruker AXS Smart 1000 X-ray diffractometer equipped with a CCD area detector and a 


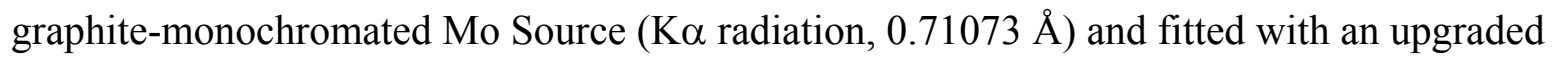
Nicolet LT-2 low temperature device. A suitable crystal of $\mathbf{1}$ was coated with paratone oil (Exxon) and mounted on a glass fiber under a stream of nitrogen at $100(2){ }^{\circ} \mathrm{C}$. The structure of 1 was solved by direct methods. $\mathrm{C}(1)$ and $\mathrm{C}(2)$ were found disordered over two positions with a refined occupancy ratio of 0.52:0.48. These two positions are shown as $\mathrm{C}(1)$ and $\mathrm{C}(1 \mathrm{~A})$ for atom $\mathrm{C}(1)$ and $\mathrm{C}(2)$ and $\mathrm{C}(2 \mathrm{~A})$ for atom $\mathrm{C}(2)$ respectively. $\mathrm{C}(1 \mathrm{~A})$ and $\mathrm{C}(2 \mathrm{~A})$ with 0.48 occupancy were deleted in the ORTEP for the clearance. Non-hydrogen atoms were anistropically refined. All $\mathrm{H}$ atoms in $\mathbf{1}$ were treated as idealized contributions. Global refinements for the unit cells and data reductions of the data sets for $\mathbf{1}$ were conducted with the Saint program. The SHELXTL (Version 5.1) proprietary software package was used for all structure solution and refinement calculations. 
Table S1. Crystal Data for 1

\begin{tabular}{|c|c|c|}
\hline Empirical formula & \multicolumn{2}{|c|}{$\mathrm{C}_{80} \mathrm{H}_{184} \mathrm{~K}_{2} \mathrm{O}_{12} \mathrm{Si}_{18} \mathrm{Zn}_{2}$} \\
\hline Formula weight & \multicolumn{2}{|l|}{2052.84} \\
\hline Temperature & \multicolumn{2}{|l|}{$-100(2){ }^{\circ} \mathrm{C}$} \\
\hline Wavelength & \multicolumn{2}{|l|}{$0.71073 \AA$} \\
\hline Crystal system & \multicolumn{2}{|l|}{ Triclinic } \\
\hline Space group & \multicolumn{2}{|l|}{$P-1$} \\
\hline \multirow[t]{3}{*}{ Unit cell dimensions } & $a=12.603(2) \AA$ & $\alpha=60.196(3)^{\circ}$ \\
\hline & $b=16.901(3) \AA$ & $\beta=75.945(3)^{\circ}$ \\
\hline & $c=16.917(3) \AA$ & $\gamma=78.761(3)^{\circ}$ \\
\hline Volume & \multicolumn{2}{|l|}{$3021.3(9) \AA^{3}$} \\
\hline$Z$ & \multicolumn{2}{|l|}{1} \\
\hline Density (calculated) & \multicolumn{2}{|l|}{$1.128 \mathrm{~g} / \mathrm{cm}^{3}$} \\
\hline Absorption coefficient & \multicolumn{2}{|l|}{$0.689 \mathrm{~mm}^{-1}$} \\
\hline$F(000)$ & \multicolumn{2}{|l|}{1110} \\
\hline Crystal size & \multicolumn{2}{|c|}{$0.40 \times 0.40 \times 0.30 \mathrm{~mm}^{3}$} \\
\hline$\theta$ range for data collection & \multicolumn{2}{|l|}{1.39 to $28.27^{\circ}$} \\
\hline Index ranges & \multicolumn{2}{|c|}{$-16 \leq h \leq 16,-22 \leq k \leq 22,-21 \leq l \leq 22$} \\
\hline Reflections collected & \multicolumn{2}{|c|}{32547} \\
\hline Independent reflections & \multicolumn{2}{|c|}{$14040[R(\mathrm{int})=0.0385]$} \\
\hline Completeness to $\theta=28.27^{\circ}$ & \multicolumn{2}{|l|}{$93.6 \%$} \\
\hline Max. and min. transmission & \multicolumn{2}{|c|}{0.8199 and 0.7701} \\
\hline Refinement method & \multicolumn{2}{|c|}{ Full-matrix least-squares on $F^{2}$} \\
\hline Data / restraints / parameters & \multicolumn{2}{|c|}{$14040 / 0 / 552$} \\
\hline Goodness-of-fit on $F^{2}$ & \multicolumn{2}{|l|}{1.014} \\
\hline Final $R$ indices $[I>2 \sigma(I)]$ & \multicolumn{2}{|c|}{$R 1=0.0466, \mathrm{w} R 2=0.1059$} \\
\hline$R$ indices (all data) & \multicolumn{2}{|c|}{$R 1=0.0779, \mathrm{w} R 2=0.1223$} \\
\hline Largest diff. peak and hole & \multicolumn{2}{|c|}{0.917 and -0.952 e..$\AA^{-3}$} \\
\hline
\end{tabular}


Table S2. Atomic Coordinates $\left(\times 10^{4}\right)$ and Equivalent Isotropic Displacement Parameters $\left(\AA^{2} \times\right.$ $10^{3}$ ) in 1. U(eq) is Defined as One Third of the Trace of the Orthogonalized $\mathrm{U}^{\mathrm{ij}}$ Tensor.

\begin{tabular}{|c|c|c|c|c|}
\hline & $\mathrm{X}$ & $\mathrm{y}$ & z & $\mathrm{U}(\mathrm{eq})$ \\
\hline $\mathrm{C}(1)$ & $6662(6)$ & $7609(5)$ & $4968(3)$ & $37(2)$ \\
\hline $\mathrm{C}(2)$ & $7796(6)$ & $7741(5)$ & $5010(4)$ & $37(2)$ \\
\hline $\mathrm{C}(3)$ & $9806(2)$ & $9770(2)$ & $526(2)$ & $38(1)$ \\
\hline $\mathrm{C}(4)$ & $4330(3)$ & $7515(3)$ & $3139(3)$ & $73(1)$ \\
\hline$C(5)$ & $4033(4)$ & $7082(3)$ & $5140(3)$ & $97(2)$ \\
\hline$C(6)$ & $4417(5)$ & 8992(3) & $3633(3)$ & $119(2)$ \\
\hline$C(7)$ & $6946(3)$ & $5617(2)$ & $3359(2)$ & $57(1)$ \\
\hline $\mathrm{C}(8)$ & $6359(3)$ & $5102(2)$ & $5384(2)$ & $55(1)$ \\
\hline $\mathrm{C}(9)$ & $8636(3)$ & $5561(3)$ & $4399(3)$ & $79(1)$ \\
\hline$C(10)$ & $10696(5)$ & $7592(3)$ & $3319(4)$ & $112(2)$ \\
\hline $\mathrm{C}(11)$ & $10570(4)$ & $7744(3)$ & $5043(3)$ & $90(1)$ \\
\hline$C(12)$ & $11063(3)$ & $9385(2)$ & $3156(2)$ & $62(1)$ \\
\hline$C(13)$ & $6386(3)$ & $10480(4)$ & $4055(3)$ & $106(2)$ \\
\hline$C(14)$ & $8455(3)$ & $10369(3)$ & $4680(3)$ & $72(1)$ \\
\hline$C(15)$ & $8461(3)$ & $11180(3)$ & $2650(2)$ & $76(1)$ \\
\hline$C(16)$ & $6841(3)$ & $7865(2)$ & $984(2)$ & $53(1)$ \\
\hline$C(17)$ & $9181(3)$ & $7162(2)$ & $1352(2)$ & $56(1)$ \\
\hline$C(18)$ & $8756(3)$ & $8407(2)$ & $-596(2)$ & $62(1)$ \\
\hline C(19) & $7094(3)$ & $10842(3)$ & $-985(2)$ & $73(1)$ \\
\hline$C(20)$ & $5663(3)$ & $10276(3)$ & $879(2)$ & $62(1)$ \\
\hline $\mathrm{C}(21)$ & $7365(3)$ & $11608(2)$ & $227(3)$ & $72(1)$ \\
\hline $\mathrm{C}(22)$ & $120(3)$ & $4197(2)$ & $2425(2)$ & $58(1)$ \\
\hline$C(23)$ & $336(3)$ & $4746(2)$ & $2819(2)$ & $56(1)$ \\
\hline$C(24)$ & $1628(3)$ & $5641(2)$ & $2715(2)$ & $55(1)$ \\
\hline$C(25)$ & $2718(3)$ & 5991(2) & $2204(2)$ & $58(1)$ \\
\hline$C(26)$ & 4601(3) & $5510(2)$ & $1945(2)$ & $61(1)$ \\
\hline $\mathrm{C}(27)$ & $5408(3)$ & $4687(3)$ & $2257(2)$ & $62(1)$ \\
\hline $\mathrm{C}(28)$ & $5957(3)$ & $3280(3)$ & $2242(3)$ & $67(1)$ \\
\hline C(29) & $5706(3)$ & $2684(3)$ & 1903(3) & $66(1)$ \\
\hline$C(30)$ & $4404(3)$ & $1725(2)$ & $2135(3)$ & $68(1)$ \\
\hline$C(31)$ & $3298(3)$ & $1418(2)$ & $2655(3)$ & $69(1)$ \\
\hline$C(32)$ & $1423(3)$ & $1954(2)$ & 2751(3) & $63(1)$ \\
\hline$C(33)$ & $670(3)$ & $2799(2)$ & $2379(3)$ & $61(1)$ \\
\hline$C(34)$ & $8026(3)$ & $4454(2)$ & $108(2)$ & $56(1)$ \\
\hline$C(35)$ & $8412(3)$ & 4973(3) & $373(3)$ & $67(1)$ \\
\hline$C(36)$ & $7887(5)$ & $5788(3)$ & 283(3) & $90(1)$ \\
\hline$C(37)$ & $6968(5)$ & $6117(3)$ & $-64(3)$ & $94(2)$ \\
\hline$C(38)$ & $6533(4)$ & $5638(4)$ & $-342(3)$ & $85(1)$ \\
\hline C(39) & $7057(3)$ & 4798(3) & $-258(2)$ & $69(1)$ \\
\hline$C(40)$ & $8605(4)$ & $3580(3)$ & $194(4)$ & $108(2)$ \\
\hline$C(1 \mathrm{~A})$ & $7303(6)$ & $7225(5)$ & $4986(4)$ & $31(2)$ \\
\hline
\end{tabular}




\begin{tabular}{lrlll}
$\mathrm{C}(2 \mathrm{~A})$ & $7325(7)$ & $8156(6)$ & $4948(4)$ & $35(2)$ \\
$\mathrm{K}(1)$ & $2995(1)$ & $3591(1)$ & $2634(1)$ & $43(1)$ \\
$\mathrm{O}(1)$ & $867(2)$ & $3400(1)$ & $2676(1)$ & $48(1)$ \\
$\mathrm{O}(2)$ & $1389(2)$ & $5076(1)$ & $2393(1)$ & $48(1)$ \\
$\mathrm{O}(3)$ & $3531(2)$ & $5236(1)$ & $2386(1)$ & $49(1)$ \\
$\mathrm{O}(4)$ & $5180(2)$ & $4062(2)$ & $2003(1)$ & $53(1)$ \\
$\mathrm{O}(5)$ & $4657(2)$ & $2365(2)$ & $2362(2)$ & $57(1)$ \\
$\mathrm{O}(6)$ & $2517(2)$ & $2190(1)$ & $2365(2)$ & $56(1)$ \\
$\mathrm{Si}(1)$ & $6732(1)$ & $7438(1)$ & $3880(1)$ & $54(1)$ \\
$\mathrm{Si}(2)$ & $8353(1)$ & $8826(1)$ & $3832(1)$ & $74(1)$ \\
$\mathrm{Si}(3)$ & $8367(1)$ & $9337(1)$ & $992(1)$ & $31(1)$ \\
$\mathrm{Si}(4)$ & $4834(1)$ & $7750(1)$ & $3948(1)$ & $65(1)$ \\
$\mathrm{Si}(5)$ & $7152(1)$ & $5901(1)$ & $4249(1)$ & $45(1)$ \\
$\mathrm{Si}(6)$ & $10220(1)$ & $8384(1)$ & $3850(1)$ & $64(1)$ \\
$\mathrm{Si}(7)$ & $7924(1)$ & $10236(1)$ & $3816(1)$ & $59(1)$ \\
$\mathrm{Si}(8)$ & $8289(1)$ & $8187(1)$ & $633(1)$ & $37(1)$ \\
$\mathrm{Si}(9)$ & $7097(1)$ & $10542(1)$ & $240(1)$ & $40(1)$ \\
$\mathrm{Zn}(1)$ & $7834(1)$ & $8651(1)$ & $2647(1)$ & $30(1)$ \\
& & & & \\
\hline
\end{tabular}


Table S3. Bond Distances $(\AA)$ in $\mathbf{1 .}$

\begin{tabular}{llll}
\hline $\mathrm{C}(1)-\mathrm{C}(2)$ & $1.513(10)$ & $\mathrm{C}(1)-\mathrm{Si}(1)$ & $1.981(5)$ \\
$\mathrm{C}(2)-\mathrm{Si}(2)$ & $2.004(6)$ & $\mathrm{C}(2)-\mathrm{K}(1) \# 1$ & $3.460(6)$ \\
$\mathrm{C}(3)-\mathrm{C}(3) \# 2$ & $1.536(5)$ & $\mathrm{C}(3)-\mathrm{Si}(3)$ & $1.923(3)$ \\
$\mathrm{C}(4)-\mathrm{Si}(4)$ & $1.869(4)$ & $\mathrm{C}(5)-\mathrm{Si}(4)$ & $1.885(4)$ \\
$\mathrm{C}(6)-\mathrm{Si}(4)$ & $1.887(4)$ & $\mathrm{C}(7)-\mathrm{Si}(5)$ & $1.877(3)$ \\
$\mathrm{C}(8)-\mathrm{Si}(5)$ & $1.878(3)$ & $\mathrm{C}(9)-\mathrm{Si}(5)$ & $1.887(3)$ \\
$\mathrm{C}(10)-\mathrm{Si}(6)$ & $1.891(4)$ & $\mathrm{C}(11)-\mathrm{Si}(6)$ & $1.875(4)$ \\
$\mathrm{C}(12)-\mathrm{Si}(6)$ & $1.868(3)$ & $\mathrm{C}(13)-\mathrm{Si}(7)$ & $1.889(4)$ \\
$\mathrm{C}(14)-\mathrm{Si}(7)$ & $1.871(4)$ & $\mathrm{C}(15)-\mathrm{Si}(7)$ & $1.881(4)$ \\
$\mathrm{C}(16)-\mathrm{Si}(8)$ & $1.877(3)$ & $\mathrm{C}(17)-\mathrm{Si}(8)$ & $1.885(3)$ \\
$\mathrm{C}(18)-\mathrm{Si}(8)$ & $1.877(3)$ & $\mathrm{C}(19)-\mathrm{Si}(9)$ & $1.873(3)$ \\
$\mathrm{C}(20)-\mathrm{Si}(9)$ & $1.876(3)$ & $\mathrm{C}(21)-\mathrm{Si}(9)$ & $1.885(4)$ \\
$\mathrm{C}(22)-\mathrm{O}(1)$ & $1.418(4)$ & $\mathrm{C}(22)-\mathrm{C}(23)$ & $1.477(5)$ \\
$\mathrm{C}(23)-\mathrm{O}(2)$ & $1.420(4)$ & $\mathrm{C}(24)-\mathrm{O}(2)$ & $1.418(4)$ \\
$\mathrm{C}(24)-\mathrm{C}(25)$ & $1.488(5)$ & $\mathrm{C}(25)-\mathrm{O}(3)$ & $1.423(4)$ \\
$\mathrm{C}(26)-\mathrm{O}(3)$ & $1.414(4)$ & $\mathrm{C}(26)-\mathrm{C}(27)$ & $1.494(5)$ \\
$\mathrm{C}(27)-\mathrm{O}(4)$ & $1.419(4)$ & $\mathrm{C}(28)-\mathrm{O}(4)$ & $1.423(4)$ \\
$\mathrm{C}(28)-\mathrm{C}(29)$ & $1.491(5)$ & $\mathrm{C}(29)-\mathrm{O}(5)$ & $1.416(4)$ \\
$\mathrm{C}(30)-\mathrm{O}(5)$ & $1.424(4)$ & $\mathrm{C}(30)-\mathrm{C}(31)$ & $1.489(5)$ \\
$\mathrm{C}(31)-\mathrm{O}(6)$ & $1.418(4)$ & $\mathrm{C}(32)-\mathrm{O}(6)$ & $1.416(4)$ \\
$\mathrm{C}(32)-\mathrm{C}(33)$ & $1.486(5)$ & $\mathrm{C}(33)-\mathrm{O}(1)$ & $1.418(4)$ \\
$\mathrm{C}(34)-\mathrm{C}(35)$ & $1.367(5)$ & $\mathrm{C}(34)-\mathrm{C}(39)$ & $1.395(5)$ \\
$\mathrm{C}(34)-\mathrm{C}(40)$ & $1.468(5)$ & $\mathrm{C}(35)-\mathrm{C}(36)$ & $1.357(6)$ \\
$\mathrm{C}(36)-\mathrm{C}(37)$ & $1.323(7)$ & $\mathrm{C}(37)-\mathrm{C}(38)$ & $1.362(7)$ \\
$\mathrm{C}(38)-\mathrm{C}(39)$ & $1.397(6)$ & $\mathrm{C}(38)-\mathrm{K}(1) \# 3$ & $3.354(4)$ \\
$\mathrm{C}(1 \mathrm{~A})-\mathrm{C}(2 \mathrm{~A})$ & $1.548(11)$ & $\mathrm{C}(1 \mathrm{~A})-\mathrm{Si}(1)$ & $2.008(5)$ \\
$\mathrm{C}(1 \mathrm{~A})-\mathrm{K}(1) \# 1$ & $3.494(5)$ & $\mathrm{C}(2 \mathrm{~A})-\mathrm{Si}(2)$ & $1.945(6)$ \\
$\mathrm{K}(1)-\mathrm{O}(1)$ & $2.742(2)$ & $\mathrm{K}(1)-\mathrm{O}(5)$ & $2.772(2)$ \\
$\mathrm{K}(1)-\mathrm{O}(3)$ & $2.793(2)$ & $\mathrm{Si}(3)-\mathrm{Zn}(1)$ & $2.4076(8)$ \\
$\mathrm{K}(1)-\mathrm{O}(6)$ & $2.818(2)$ & & \\
& & &
\end{tabular}

Symmetry transformations used to generate equivalent atoms:

$\# 1 \quad-x+1,-y+1,-z+1 \quad \# 2 \quad-x+2,-y+2,-z \quad \# 3 \quad-x+1,-y+1,-z$ 
Table S4. Bond Angles (deg) in 1.

\begin{tabular}{|c|c|c|c|}
\hline$\overline{C(2)-C(1)-S i(1)}$ & $109.5(5)$ & $C(1)-C(2)-S i(2)$ & $108.7(5)$ \\
\hline $\mathrm{C}(1)-\mathrm{C}(2)-\mathrm{K}(1) \# 1$ & $83.6(3)$ & $\mathrm{Si}(2)-\mathrm{C}(2)-\mathrm{K}(1) \# 1$ & $157.6(3)$ \\
\hline $\mathrm{C}(3) \# 2-\mathrm{C}(3)-\mathrm{Si}(3)$ & $117.7(2)$ & $\mathrm{O}(1)-\mathrm{C}(22)-\mathrm{C}(23)$ & $109.0(3)$ \\
\hline $\mathrm{O}(2)-\mathrm{C}(23)-\mathrm{C}(22)$ & $109.1(3)$ & $\mathrm{O}(2)-\mathrm{C}(24)-\mathrm{C}(25)$ & $108.4(2)$ \\
\hline $\mathrm{O}(3)-\mathrm{C}(25)-\mathrm{C}(24)$ & $109.0(3)$ & $\mathrm{O}(3)-\mathrm{C}(26)-\mathrm{C}(27)$ & $108.9(3)$ \\
\hline $\mathrm{O}(4)-\mathrm{C}(27)-\mathrm{C}(26)$ & $109.2(3)$ & $\mathrm{O}(4)-\mathrm{C}(28)-\mathrm{C}(29)$ & $108.8(3)$ \\
\hline $\mathrm{O}(5)-\mathrm{C}(29)-\mathrm{C}(28)$ & $109.3(3)$ & $\mathrm{O}(5)-\mathrm{C}(30)-\mathrm{C}(31)$ & $108.8(3)$ \\
\hline $\mathrm{O}(6)-\mathrm{C}(31)-\mathrm{C}(30)$ & $108.3(3)$ & $\mathrm{O}(6)-\mathrm{C}(32)-\mathrm{C}(33)$ & $108.4(3)$ \\
\hline $\mathrm{O}(1)-\mathrm{C}(33)-\mathrm{C}(32)$ & $108.4(3)$ & $C(35)-C(34)-C(39)$ & $116.9(4)$ \\
\hline$C(35)-C(34)-C(40)$ & $122.0(4)$ & $C(39)-C(34)-C(40)$ & $121.2(4)$ \\
\hline$C(36)-C(35)-C(34)$ & $122.1(4)$ & $C(37)-C(36)-C(35)$ & $121.3(5)$ \\
\hline$C(36)-C(37)-C(38)$ & $119.9(5)$ & $\mathrm{C}(37)-\mathrm{C}(38)-\mathrm{C}(39)$ & $120.0(4)$ \\
\hline $\mathrm{C}(37)-\mathrm{C}(38)-\mathrm{K}(1) \# 3$ & $114.8(3)$ & $\mathrm{C}(39)-\mathrm{C}(38)-\mathrm{K}(1) \# 3$ & $87.3(2)$ \\
\hline $\mathrm{C}(34)-\mathrm{C}(39)-\mathrm{C}(38)$ & $119.8(4)$ & $C(2 A)-C(1 A)-S i(1)$ & $109.4(5)$ \\
\hline$C(2 A)-C(1 A)-K(1) \# 1$ & $82.9(3)$ & $\mathrm{Si}(1)-\mathrm{C}(1 \mathrm{~A})-\mathrm{K}(1) \# 1$ & $150.8(3)$ \\
\hline C(1A)-C(2A)-Si(2) & $105.3(5)$ & $\mathrm{O}(1)-\mathrm{K}(1)-\mathrm{O}(5)$ & $119.09(7)$ \\
\hline $\mathrm{O}(1)-\mathrm{K}(1)-\mathrm{O}(3)$ & $119.63(6)$ & $\mathrm{O}(5)-\mathrm{K}(1)-\mathrm{O}(3)$ & $119.23(7)$ \\
\hline $\mathrm{O}(1)-\mathrm{K}(1)-\mathrm{O}(6)$ & $59.72(6)$ & $\mathrm{O}(5)-\mathrm{K}(1)-\mathrm{O}(6)$ & $59.39(7)$ \\
\hline $\mathrm{O}(3)-\mathrm{K}(1)-\mathrm{O}(6)$ & $164.64(7)$ & $\mathrm{O}(1)-\mathrm{K}(1)-\mathrm{O}(4)$ & $162.37(7)$ \\
\hline $\mathrm{O}(5)-\mathrm{K}(1)-\mathrm{O}(4)$ & $60.09(7)$ & $\mathrm{O}(3)-\mathrm{K}(1)-\mathrm{O}(4)$ & $59.26(7)$ \\
\hline $\mathrm{O}(6)-\mathrm{K}(1)-\mathrm{O}(4)$ & $116.09(7)$ & $\mathrm{O}(1)-\mathrm{K}(1)-\mathrm{O}(2)$ & $60.69(6)$ \\
\hline $\mathrm{O}(5)-\mathrm{K}(1)-\mathrm{O}(2)$ & $164.73(7)$ & $\mathrm{O}(3)-\mathrm{K}(1)-\mathrm{O}(2)$ & $58.94(6)$ \\
\hline $\mathrm{O}(6)-\mathrm{K}(1)-\mathrm{O}(2)$ & $117.76(7)$ & $\mathrm{O}(4)-\mathrm{K}(1)-\mathrm{O}(2)$ & $114.92(7)$ \\
\hline $\mathrm{O}(1)-\mathrm{K}(1)-\mathrm{C}(38) \# 3$ & $88.80(9)$ & $\mathrm{O}(5)-\mathrm{K}(1)-\mathrm{C}(38) \# 3$ & $75.82(9)$ \\
\hline $\mathrm{O}(3)-\mathrm{K}(1)-\mathrm{C}(38) \# 3$ & $91.10(11)$ & $\mathrm{O}(6)-\mathrm{K}(1)-\mathrm{C}(38) \# 3$ & $73.63(11)$ \\
\hline $\mathrm{O}(4)-\mathrm{K}(1)-\mathrm{C}(38) \# 3$ & $73.81(10)$ & $\mathrm{O}(2)-\mathrm{K}(1)-\mathrm{C}(38) \# 3$ & $88.96(9)$ \\
\hline $\mathrm{O}(1)-\mathrm{K}(1)-\mathrm{C}(2) \# 1$ & $80.35(12)$ & $\mathrm{O}(5)-\mathrm{K}(1)-\mathrm{C}(2) \# 1$ & $97.33(16)$ \\
\hline $\mathrm{O}(3)-\mathrm{K}(1)-\mathrm{C}(2) \# 1$ & $106.29(12)$ & $\mathrm{O}(6)-\mathrm{K}(1)-\mathrm{C}(2) \# 1$ & $88.90(12)$ \\
\hline $\mathrm{O}(4)-\mathrm{K}(1)-\mathrm{C}(2) \# 1$ & $117.22(13)$ & $\mathrm{O}(2)-\mathrm{K}(1)-\mathrm{C}(2) \# 1$ & $97.60(16)$ \\
\hline $\mathrm{C}(38) \# 3-\mathrm{K}(1)-\mathrm{C}(2) \# 1$ & $162.40(15)$ & $\mathrm{O}(1)-\mathrm{K}(1)-\mathrm{C}(1 \mathrm{~A}) \# 1$ & $95.05(12)$ \\
\hline $\mathrm{O}(5)-\mathrm{K}(1)-\mathrm{C}(1 \mathrm{~A}) \# 1$ & $101.51(15)$ & $\mathrm{O}(3)-\mathrm{K}(1)-\mathrm{C}(1 \mathrm{~A}) \# 1$ & $87.71(11)$ \\
\hline $\mathrm{O}(6)-\mathrm{K}(1)-\mathrm{C}(1 \mathrm{~A}) \# 1$ & $107.64(11)$ & $\mathrm{O}(4)-\mathrm{K}(1)-\mathrm{C}(1 \mathrm{~A}) \# 1$ & $102.39(12)$ \\
\hline $\mathrm{O}(2)-\mathrm{K}(1)-\mathrm{C}(1 \mathrm{~A}) \# 1$ & $93.63(15)$ & $\mathrm{C}(38) \# 3-\mathrm{K}(1)-\mathrm{C}(1 \mathrm{~A}) \# 1$ & $176.07(15)$ \\
\hline $\mathrm{C}(2) \# 1-\mathrm{K}(1)-\mathrm{C}(1 \mathrm{~A}) \# 1$ & $19.66(13)$ & $\mathrm{C}(22)-\mathrm{O}(1)-\mathrm{C}(33)$ & $112.8(2)$ \\
\hline $\mathrm{C}(22)-\mathrm{O}(1)-\mathrm{K}(1)$ & $117.41(18)$ & $\mathrm{C}(33)-\mathrm{O}(1)-\mathrm{K}(1)$ & $118.79(18)$ \\
\hline $\mathrm{C}(24)-\mathrm{O}(2)-\mathrm{C}(23)$ & $111.9(2)$ & $\mathrm{C}(24)-\mathrm{O}(2)-\mathrm{K}(1)$ & $112.40(17)$ \\
\hline $\mathrm{C}(23)-\mathrm{O}(2)-\mathrm{K}(1)$ & $109.74(16)$ & $\mathrm{C}(26)-\mathrm{O}(3)-\mathrm{C}(25)$ & $112.7(2)$ \\
\hline $\mathrm{C}(26)-\mathrm{O}(3)-\mathrm{K}(1)$ & $119.06(19)$ & $\mathrm{C}(25)-\mathrm{O}(3)-\mathrm{K}(1)$ & $119.49(18)$ \\
\hline $\mathrm{C}(27)-\mathrm{O}(4)-\mathrm{C}(28)$ & $111.9(3)$ & $\mathrm{C}(27)-\mathrm{O}(4)-\mathrm{K}(1)$ & $113.69(18)$ \\
\hline $\mathrm{C}(28)-\mathrm{O}(4)-\mathrm{K}(1)$ & $112.5(2)$ & $\mathrm{C}(29)-\mathrm{O}(5)-\mathrm{C}(30)$ & $112.4(3)$ \\
\hline $\mathrm{C}(29)-\mathrm{O}(5)-\mathrm{K}(1)$ & $117.78(19)$ & $\mathrm{C}(30)-\mathrm{O}(5)-\mathrm{K}(1)$ & $119.12(19)$ \\
\hline $\mathrm{C}(31)-\mathrm{O}(6)-\mathrm{C}(32)$ & $112.6(3)$ & $\mathrm{C}(31)-\mathrm{O}(6)-\mathrm{K}(1)$ & $112.2(2)$ \\
\hline $\mathrm{C}(32)-\mathrm{O}(6)-\mathrm{K}(1)$ & $112.66(18)$ & $C(1)-\operatorname{Si}(1)-C(1 A)$ & $26.9(2)$ \\
\hline C(1)-Si(1)-Si(5) & $110.8(2)$ & $C(1 \mathrm{~A})-\mathrm{Si}(1)-\operatorname{Si}(5)$ & $91.1(2)$ \\
\hline
\end{tabular}




\begin{tabular}{|c|c|c|c|}
\hline C(1)-Si(1)-Si(4) & $91.7(2)$ & $\mathrm{C}(1 \mathrm{~A})-\mathrm{Si}(1)-\mathrm{Si}(4)$ & $115.9(2)$ \\
\hline $\mathrm{Si}(5)-\mathrm{Si}(1)-\mathrm{Si}(4)$ & $107.49(4)$ & $\mathrm{C}(1)-\mathrm{Si}(1)-\mathrm{Zn}(1)$ & $102.97(15)$ \\
\hline $\mathrm{C}(1 \mathrm{~A})-\mathrm{Si}(1)-\mathrm{Zn}(1)$ & $100.62(16)$ & $\operatorname{Si}(5)-\operatorname{Si}(1)-\operatorname{Zn}(1)$ & $123.26(5)$ \\
\hline $\operatorname{Si}(4)-\operatorname{Si}(1)-\operatorname{Zn}(1)$ & $115.79(5)$ & $\mathrm{C}(2 \mathrm{~A})-\mathrm{Si}(2)-\mathrm{C}(2)$ & $23.5(2)$ \\
\hline$C(2 A)-\operatorname{Si}(2)-\operatorname{Si}(7)$ & $96.6(3)$ & $\mathrm{C}(2)-\mathrm{Si}(2)-\mathrm{Si}(7)$ & $116.1(2)$ \\
\hline$C(2 A)-\operatorname{Si}(2)-\operatorname{Si}(6)$ & $118.0(3)$ & $\mathrm{C}(2)-\mathrm{Si}(2)-\mathrm{Si}(6)$ & $97.3(3)$ \\
\hline $\operatorname{Si}(7)-\operatorname{Si}(2)-\operatorname{Si}(6)$ & $110.01(4)$ & $C(2 A)-\operatorname{Si}(2)-Z n(1)$ & $104.86(19)$ \\
\hline $\mathrm{C}(2)-\mathrm{Si}(2)-\mathrm{Zn}(1)$ & 103.92(17) & $\operatorname{Si}(7)-\operatorname{Si}(2)-Z n(1)$ & $118.39(6)$ \\
\hline $\operatorname{Si}(6)-\operatorname{Si}(2)-\operatorname{Zn}(1)$ & $108.96(5)$ & $\mathrm{C}(3)-\mathrm{Si}(3)-\mathrm{Si}(8)$ & 109.97(10) \\
\hline $\mathrm{C}(3)-\mathrm{Si}(3)-\mathrm{Si}(9)$ & $108.60(9)$ & $\operatorname{Si}(8)-\operatorname{Si}(3)-\operatorname{Si}(9)$ & $106.84(4)$ \\
\hline $\mathrm{C}(3)-\operatorname{Si}(3)-\mathrm{Zn}(1)$ & $114.83(8)$ & $\operatorname{Si}(8)-\operatorname{Si}(3)-\operatorname{Zn}(1)$ & $104.75(3)$ \\
\hline $\operatorname{Si}(9)-\operatorname{Si}(3)-\operatorname{Zn}(1)$ & $111.53(3)$ & $\mathrm{C}(4)-\mathrm{Si}(4)-\mathrm{C}(5)$ & $107.5(2)$ \\
\hline$C(4)-\operatorname{Si}(4)-C(6)$ & $107.4(2)$ & $\mathrm{C}(5)-\mathrm{Si}(4)-\mathrm{C}(6)$ & $105.2(2)$ \\
\hline $\mathrm{C}(4)-\mathrm{Si}(4)-\mathrm{Si}(1)$ & $112.42(12)$ & $\mathrm{C}(5)-\mathrm{Si}(4)-\mathrm{Si}(1)$ & $113.30(16)$ \\
\hline $\mathrm{C}(6)-\mathrm{Si}(4)-\mathrm{Si}(1)$ & $110.6(2)$ & $\mathrm{C}(7)-\mathrm{Si}(5)-\mathrm{C}(8)$ & $106.92(15)$ \\
\hline C(7)-Si(5)-C(9) & $107.57(18)$ & $\mathrm{C}(8)-\mathrm{Si}(5)-\mathrm{C}(9)$ & $104.64(17)$ \\
\hline $\mathrm{C}(7)-\mathrm{Si}(5)-\mathrm{Si}(1)$ & $114.46(11)$ & C(8)-Si(5)-Si(1) & $113.46(12)$ \\
\hline C(9)-Si(5)-Si(1) & $109.19(15)$ & $C(12)-\operatorname{Si}(6)-C(11)$ & 108.07(19) \\
\hline$C(12)-\operatorname{Si}(6)-C(10)$ & 106.95(19) & $C(11)-\operatorname{Si}(6)-C(10)$ & $106.5(2)$ \\
\hline $\mathrm{C}(12)-\mathrm{Si}(6)-\mathrm{Si}(2)$ & $111.85(13)$ & $C(11)-\operatorname{Si}(6)-\operatorname{Si}(2)$ & $113.43(16)$ \\
\hline$C(10)-\operatorname{Si}(6)-\operatorname{Si}(2)$ & $109.7(2)$ & $C(14)-\operatorname{Si}(7)-C(15)$ & $106.08(19)$ \\
\hline$C(14)-\operatorname{Si}(7)-C(13)$ & $105.1(2)$ & $C(15)-\operatorname{Si}(7)-C(13)$ & $107.83(19)$ \\
\hline$C(14)-\operatorname{Si}(7)-\operatorname{Si}(2)$ & $116.71(13)$ & $C(15)-\operatorname{Si}(7)-\operatorname{Si}(2)$ & $110.55(15)$ \\
\hline $\mathrm{C}(13)-\mathrm{Si}(7)-\mathrm{Si}(2)$ & 110.09(19) & $C(18)-\operatorname{Si}(8)-C(16)$ & $106.96(16)$ \\
\hline C(18)-Si(8)-C(17) & $106.54(16)$ & $C(16)-\operatorname{Si}(8)-C(17)$ & $108.23(15)$ \\
\hline C(18)-Si(8)-Si(3) & $118.84(11)$ & C(16)-Si(8)-Si(3) & $108.95(10)$ \\
\hline $\mathrm{C}(17)-\mathrm{Si}(8)-\mathrm{Si}(3)$ & $106.92(12)$ & $C(19)-\operatorname{Si}(9)-C(20)$ & $106.49(17)$ \\
\hline C(19)-Si(9)-C(21) & 107.10(19) & $C(20)-\operatorname{Si}(9)-C(21)$ & $107.02(18)$ \\
\hline C(19)-Si(9)-Si(3) & $115.76(13)$ & $\mathrm{C}(20)-\mathrm{Si}(9)-\mathrm{Si}(3)$ & $110.59(12)$ \\
\hline $\mathrm{C}(21)-\mathrm{Si}(9)-\mathrm{Si}(3)$ & $109.47(13)$ & $\mathrm{Si}(3)-\mathrm{Zn}(1)-\mathrm{Si}(2)$ & $133.72(3)$ \\
\hline $\operatorname{Si}(3)-\operatorname{Zn}(1)-\operatorname{Si}(1)$ & $138.45(3)$ & $\operatorname{Si}(2)-\operatorname{Zn}(1)-\operatorname{Si}(1)$ & $87.63(3)$ \\
\hline
\end{tabular}

Symmetry transformations used to generate equivalent atoms:

$\# 1 \quad-\mathrm{x}+1,-\mathrm{y}+1,-\mathrm{z}+1 \quad \# 2 \quad-\mathrm{x}+2,-\mathrm{y}+2,-\mathrm{z} \quad \# 3 \quad-\mathrm{x}+1,-\mathrm{y}+1,-\mathrm{z}$ 
Table S5. Anisotropic Displacement Parameters $\left(\AA^{2} \times 10^{3}\right)$ in 1. The Anisotropic Displacement Factor Exponent Takes the Form: $-2 \pi^{2}\left[h^{2} a^{* 2} U^{11}+\ldots+2 h k a * b * U^{12}\right]$

\begin{tabular}{|c|c|c|c|c|c|c|}
\hline & $\mathrm{U}^{11}$ & $\mathrm{U}^{22}$ & $\mathrm{U}^{33}$ & $\mathrm{U}^{23}$ & $\mathrm{U}^{13}$ & $\mathrm{U}^{12}$ \\
\hline$\overline{C(1)}$ & $44(4)$ & $39(3)$ & $26(3)$ & $-11(2)$ & $2(2)$ & $-19(3)$ \\
\hline$C(2)$ & $45(4)$ & $39(4)$ & $26(3)$ & $-14(3)$ & $-2(2)$ & $-13(3)$ \\
\hline $\mathrm{C}(3)$ & $36(1)$ & $42(2)$ & $30(1)$ & $-11(1)$ & $-1(1)$ & $-13(1)$ \\
\hline$C(4)$ & $59(2)$ & $76(3)$ & $61(2)$ & $-18(2)$ & $-6(2)$ & $-2(2)$ \\
\hline$C(5)$ & $103(3)$ & $74(3)$ & $54(2)$ & $-7(2)$ & $25(2)$ & $7(2)$ \\
\hline$C(6)$ & $186(6)$ & $45(2)$ & $80(3)$ & $-23(2)$ & $19(3)$ & $17(3)$ \\
\hline$C(7)$ & $71(2)$ & $50(2)$ & $46(2)$ & $-18(2)$ & $-3(2)$ & $-19(2)$ \\
\hline $\mathrm{C}(8)$ & $62(2)$ & $44(2)$ & $42(2)$ & $-8(1)$ & $1(1)$ & $-15(2)$ \\
\hline$C(9)$ & $46(2)$ & 121(4) & $54(2)$ & $-33(2)$ & $-5(2)$ & $-5(2)$ \\
\hline$C(10)$ & $179(5)$ & $73(3)$ & $102(4)$ & $-64(3)$ & $22(3)$ & $-46(3)$ \\
\hline$C(11)$ & 119(4) & $73(3)$ & $54(2)$ & $-10(2)$ & $-2(2)$ & $-32(3)$ \\
\hline$C(12)$ & $80(2)$ & $48(2)$ & $53(2)$ & $-23(2)$ & $11(2)$ & $-25(2)$ \\
\hline$C(13)$ & $48(2)$ & $165(5)$ & $67(3)$ & $-24(3)$ & $-2(2)$ & $-30(3)$ \\
\hline$C(14)$ & $93(3)$ & $71(3)$ & $69(2)$ & $-41(2)$ & $-29(2)$ & $-1(2)$ \\
\hline$C(15)$ & $56(2)$ & $96(3)$ & $53(2)$ & $-15(2)$ & $-2(2)$ & $-22(2)$ \\
\hline$C(16)$ & $57(2)$ & $48(2)$ & $61(2)$ & $-31(2)$ & $-5(2)$ & $-14(2)$ \\
\hline $\mathrm{C}(17)$ & $61(2)$ & $41(2)$ & $58(2)$ & $-21(2)$ & $-6(2)$ & $3(2)$ \\
\hline $\mathrm{C}(18)$ & $99(3)$ & $49(2)$ & $39(2)$ & $-23(2)$ & $7(2)$ & $-22(2)$ \\
\hline$C(19)$ & $66(2)$ & $98(3)$ & $38(2)$ & $-21(2)$ & $-18(2)$ & $7(2)$ \\
\hline $\mathrm{C}(20)$ & $49(2)$ & $68(2)$ & $62(2)$ & $-30(2)$ & $-3(2)$ & $2(2)$ \\
\hline $\mathrm{C}(21)$ & $92(3)$ & $35(2)$ & $82(3)$ & $-21(2)$ & $-19(2)$ & $-2(2)$ \\
\hline $\mathrm{C}(22)$ & $50(2)$ & $53(2)$ & $60(2)$ & $-19(2)$ & $-16(2)$ & $3(2)$ \\
\hline $\mathrm{C}(23)$ & $53(2)$ & $43(2)$ & $55(2)$ & $-18(2)$ & $-5(2)$ & $10(1)$ \\
\hline $\mathrm{C}(24)$ & $78(2)$ & $41(2)$ & $51(2)$ & $-29(2)$ & $-13(2)$ & $12(2)$ \\
\hline $\mathrm{C}(25)$ & $90(3)$ & $33(2)$ & $54(2)$ & $-23(2)$ & $-20(2)$ & $0(2)$ \\
\hline$C(26)$ & $76(2)$ & $53(2)$ & $53(2)$ & $-18(2)$ & $-13(2)$ & $-22(2)$ \\
\hline$C(27)$ & $62(2)$ & $70(2)$ & $55(2)$ & $-23(2)$ & $-15(2)$ & $-19(2)$ \\
\hline $\mathrm{C}(28)$ & $47(2)$ & $85(3)$ & $61(2)$ & $-33(2)$ & $-13(2)$ & $7(2)$ \\
\hline$C(29)$ & $58(2)$ & $75(3)$ & $60(2)$ & $-35(2)$ & $-9(2)$ & $18(2)$ \\
\hline$C(30)$ & $86(3)$ & $50(2)$ & $74(3)$ & $-40(2)$ & $-21(2)$ & $24(2)$ \\
\hline$C(31)$ & $95(3)$ & $33(2)$ & $78(3)$ & $-28(2)$ & $-21(2)$ & $6(2)$ \\
\hline$C(32)$ & $83(2)$ & $43(2)$ & $68(2)$ & $-27(2)$ & $-7(2)$ & $-20(2)$ \\
\hline $\mathrm{C}(33)$ & $64(2)$ & $61(2)$ & $67(2)$ & $-32(2)$ & $-14(2)$ & $-14(2)$ \\
\hline$C(34)$ & $65(2)$ & $53(2)$ & $42(2)$ & $-21(2)$ & $4(2)$ & $-10(2)$ \\
\hline$C(35)$ & $65(2)$ & $80(3)$ & $61(2)$ & $-33(2)$ & $-13(2)$ & $-16(2)$ \\
\hline$C(36)$ & $114(4)$ & $78(3)$ & $86(3)$ & $-40(3)$ & $-3(3)$ & $-37(3)$ \\
\hline$C(37)$ & $141(5)$ & $64(3)$ & $58(3)$ & $-23(2)$ & $-2(3)$ & $-4(3)$ \\
\hline $\mathrm{C}(38)$ & $73(3)$ & $117(4)$ & $39(2)$ & $-26(2)$ & $-10(2)$ & $19(3)$ \\
\hline$C(39)$ & $73(2)$ & 101(3) & $47(2)$ & $-41(2)$ & $1(2)$ & $-32(2)$ \\
\hline$C(40)$ & $130(4)$ & $69(3)$ & $95(4)$ & $-38(3)$ & $18(3)$ & $5(3)$ \\
\hline$C(1 A)$ & $38(4)$ & $30(3)$ & $21(3)$ & $-7(2)$ & $-3(2)$ & $-12(3)$ \\
\hline
\end{tabular}




$\begin{array}{lllllll}\mathrm{C}(2 \mathrm{~A}) & 41(4) & 39(4) & 28(3) & -20(3) & 0(3) & -8(3) \\ \mathrm{K}(1) & 47(1) & 34(1) & 45(1) & -20(1) & -4(1) & -1(1) \\ \mathrm{O}(1) & 53(1) & 41(1) & 48(1) & -19(1) & -11(1) & -3(1) \\ \mathrm{O}(2) & 60(1) & 37(1) & 45(1) & -22(1) & -6(1) & 3(1) \\ \mathrm{O}(3) & 64(1) & 39(1) & 41(1) & -16(1) & -5(1) & -9(1) \\ \mathrm{O}(4) & 50(1) & 60(1) & 47(1) & -23(1) & -8(1) & -5(1) \\ \mathrm{O}(5) & 60(1) & 52(1) & 59(1) & -32(1) & -7(1) & 11(1) \\ \mathrm{O}(6) & 71(2) & 33(1) & 60(1) & -21(1) & -12(1) & 0(1) \\ \mathrm{Si}(1) & 91(1) & 50(1) & 25(1) & -11(1) & -3(1) & -45(1) \\ \mathrm{Si}(2) & 105(1) & 105(1) & 30(1) & -31(1) & 16(1) & -84(1) \\ \mathrm{Si}(3) & 34(1) & 30(1) & 24(1) & -10(1) & -1(1) & -9(1) \\ \mathrm{Si}(4) & 95(1) & 33(1) & 38(1) & -8(1) & 15(1) & -1(1) \\ \mathrm{Si}(5) & 42(1) & 50(1) & 32(1) & -9(1) & -4(1) & -14(1) \\ \mathrm{Si}(6) & 111(1) & 45(1) & 39(1) & -22(1) & 12(1) & -40(1) \\ \mathrm{Si}(7) & 45(1) & 94(1) & 37(1) & -24(1) & -3(1) & -26(1) \\ \mathrm{Si}(8) & 46(1) & 31(1) & 29(1) & -13(1) & 1(1) & -8(1) \\ \mathrm{Si}(9) & 44(1) & 37(1) & 32(1) & -12(1) & -7(1) & -2(1) \\ \mathrm{Zn}(1) & 33(1) & 31(1) & 24(1) & -12(1) & -1(1) & -8(1) \\ & & & & & & \end{array}$


Table S6. Hydrogen Coordinates $\left(\times 10^{4}\right)$ and Isotropic Displacement Parameters $\left(\AA^{2} \times 10^{3}\right)$ in $\mathbf{1}$.

\begin{tabular}{|c|c|c|c|c|}
\hline & $\mathrm{X}$ & $\mathrm{y}$ & z & $\mathrm{U}(\mathrm{eq})$ \\
\hline $\mathrm{H}(1 \mathrm{~A})$ & 6152 & 8151 & 4921 & 45 \\
\hline $\mathrm{H}(1 \mathrm{~B})$ & 6382 & 7066 & 5540 & 45 \\
\hline $\mathrm{H}(2 \mathrm{~A})$ & 8298 & 7187 & 5090 & 44 \\
\hline $\mathrm{H}(2 \mathrm{~B})$ & 7764 & 7844 & 5544 & 44 \\
\hline $\mathrm{H}(3 \mathrm{~A})$ & 9834 & 10211 & 742 & 46 \\
\hline $\mathrm{H}(1 \mathrm{~A})$ & 6152 & 8151 & 4921 & 45 \\
\hline $\mathrm{H}(1 \mathrm{~B})$ & 6382 & 7066 & 5540 & 45 \\
\hline $\mathrm{H}(2 \mathrm{~A})$ & 8298 & 7187 & 5090 & 44 \\
\hline $\mathrm{H}(2 \mathrm{~B})$ & 7764 & 7844 & 5544 & 44 \\
\hline $\mathrm{H}(3 \mathrm{~A})$ & 9834 & 10211 & 742 & 46 \\
\hline $\mathrm{H}(3 \mathrm{~B})$ & 10343 & 9243 & 812 & 46 \\
\hline $\mathrm{H}(4 \mathrm{~A})$ & 4521 & 6870 & 3293 & 110 \\
\hline $\mathrm{H}(4 \mathrm{~B})$ & 4675 & 7903 & 2502 & 110 \\
\hline $\mathrm{H}(4 \mathrm{C})$ & 3531 & 7648 & 3197 & 110 \\
\hline $\mathrm{H}(5 \mathrm{~A})$ & 3252 & 7296 & 5142 & 146 \\
\hline $\mathrm{H}(5 \mathrm{~B})$ & 4297 & 7174 & 5586 & 146 \\
\hline $\mathrm{H}(5 \mathrm{C})$ & 4133 & 6430 & 5313 & 146 \\
\hline $\mathrm{H}(6 \mathrm{~A})$ & 4680 & 9377 & 2974 & 178 \\
\hline $\mathrm{H}(6 \mathrm{~B})$ & 4740 & 9156 & 3999 & 178 \\
\hline $\mathrm{H}(6 \mathrm{C})$ & 3615 & 9089 & 3764 & 178 \\
\hline $\mathrm{H}(7 \mathrm{~A})$ & 7198 & 4973 & 3539 & 85 \\
\hline $\mathrm{H}(7 \mathrm{~B})$ & 7368 & 6007 & 2757 & 85 \\
\hline $\mathrm{H}(7 \mathrm{C})$ & 6165 & 5722 & 3316 & 85 \\
\hline $\mathrm{H}(8 \mathrm{~A})$ & 5581 & 5188 & 5337 & 83 \\
\hline $\mathrm{H}(8 \mathrm{~B})$ & 6441 & 5232 & 5869 & 83 \\
\hline $\mathrm{H}(8 \mathrm{C})$ & 6642 & 4468 & 5540 & 83 \\
\hline $\mathrm{H}(9 \mathrm{~A})$ & 8767 & 5693 & 4868 & 118 \\
\hline $\mathrm{H}(9 \mathrm{~B})$ & 9110 & 5909 & 3810 & 118 \\
\hline $\mathrm{H}(9 \mathrm{C})$ & 8803 & 4905 & 4598 & 118 \\
\hline $\mathrm{H}(10 \mathrm{~A})$ & 10568 & 7910 & 2679 & 168 \\
\hline $\mathrm{H}(10 \mathrm{~B})$ & 10284 & 7048 & 3675 & 168 \\
\hline $\mathrm{H}(10 \mathrm{C})$ & 11481 & 7407 & 3329 & 168 \\
\hline $\mathrm{H}(11 \mathrm{~A})$ & 11346 & 7510 & 5011 & 135 \\
\hline $\mathrm{H}(11 \mathrm{~B})$ & 10112 & 7231 & 5429 & 135 \\
\hline $\mathrm{H}(11 \mathrm{C})$ & 10434 & 8157 & 5315 & 135 \\
\hline $\mathrm{H}(12 \mathrm{~A})$ & 10918 & 9768 & 3461 & 93 \\
\hline $\mathrm{H}(12 \mathrm{~B})$ & 10870 & 9745 & 2536 & 93 \\
\hline $\mathrm{H}(12 \mathrm{C})$ & 11844 & 9166 & 3105 & 93 \\
\hline $\mathrm{H}(13 \mathrm{~A})$ & 6210 & 11113 & 3949 & 158 \\
\hline $\mathrm{H}(13 \mathrm{~B})$ & 6100 & 10060 & 4699 & 158 \\
\hline $\mathrm{H}(13 \mathrm{C})$ & 6048 & 10395 & 3641 & 158 \\
\hline $\mathrm{H}(14 \mathrm{~A})$ & 9259 & 10279 & 4576 & 108 \\
\hline
\end{tabular}




\begin{tabular}{|c|c|c|c|c|}
\hline $\mathrm{H}(14 \mathrm{~B})$ & 8162 & 9914 & 5306 & 108 \\
\hline $\mathrm{H}(14 \mathrm{C})$ & 8223 & 10986 & 4611 & 108 \\
\hline $\mathrm{H}(15 \mathrm{~A})$ & 8233 & 11772 & 2643 & 114 \\
\hline $\mathrm{H}(15 \mathrm{~B})$ & 8164 & 11168 & 2172 & 114 \\
\hline $\mathrm{H}(15 \mathrm{C})$ & 9264 & 11093 & 2527 & 114 \\
\hline $\mathrm{H}(16 \mathrm{~A})$ & 6379 & 8364 & 569 & 79 \\
\hline $\mathrm{H}(16 \mathrm{~B})$ & 6561 & 7756 & 1621 & 79 \\
\hline $\mathrm{H}(16 \mathrm{C})$ & 6824 & 7308 & 946 & 79 \\
\hline $\mathrm{H}(17 \mathrm{~A})$ & 9096 & 6637 & 1282 & 84 \\
\hline $\mathrm{H}(17 \mathrm{~B})$ & 8960 & 7015 & 2003 & 84 \\
\hline $\mathrm{H}(17 \mathrm{C})$ & 9951 & 7300 & 1144 & 84 \\
\hline $\mathrm{H}(18 \mathrm{~A})$ & 8711 & 7854 & -633 & 93 \\
\hline $\mathrm{H}(18 \mathrm{~B})$ & 9516 & 8569 & -803 & 93 \\
\hline $\mathrm{H}(18 \mathrm{C})$ & 8282 & 8912 & -992 & 93 \\
\hline $\mathrm{H}(19 \mathrm{~A})$ & 6918 & 10312 & -1003 & 109 \\
\hline H(19B) & 7821 & 11017 & -1355 & 109 \\
\hline $\mathrm{H}(19 \mathrm{C})$ & 6542 & 11356 & -1239 & 109 \\
\hline $\mathrm{H}(20 \mathrm{~A})$ & 5144 & 10795 & 564 & 93 \\
\hline $\mathrm{H}(20 \mathrm{~B})$ & 5607 & 10168 & 1512 & 93 \\
\hline $\mathrm{H}(20 \mathrm{C})$ & 5489 & 9728 & 894 & 93 \\
\hline $\mathrm{H}(21 \mathrm{~A})$ & 8096 & 11786 & -128 & 108 \\
\hline $\mathrm{H}(21 \mathrm{~B})$ & 7327 & 11487 & 862 & 108 \\
\hline $\mathrm{H}(21 \mathrm{C})$ & 6810 & 12104 & -61 & 108 \\
\hline $\mathrm{H}(22 \mathrm{~A})$ & -643 & 4022 & 2664 & 69 \\
\hline $\mathrm{H}(22 \mathrm{~B})$ & 212 & 4561 & 1743 & 69 \\
\hline $\mathrm{H}(23 \mathrm{~A})$ & -232 & 5267 & 2711 & 67 \\
\hline $\mathrm{H}(23 \mathrm{~B})$ & 309 & 4364 & 3495 & 67 \\
\hline $\mathrm{H}(24 \mathrm{~A})$ & 1642 & 5285 & 3387 & 66 \\
\hline $\mathrm{H}(24 \mathrm{~B})$ & 1055 & 6159 & 2608 & 66 \\
\hline $\mathrm{H}(25 \mathrm{~A})$ & 2703 & 6344 & 1532 & 69 \\
\hline $\mathrm{H}(25 \mathrm{~B})$ & 2894 & 6402 & 2406 & 69 \\
\hline$H(26 A)$ & 4764 & 5958 & 2106 & 73 \\
\hline $\mathrm{H}(26 \mathrm{~B})$ & 4653 & 5806 & 1266 & 73 \\
\hline $\mathrm{H}(27 \mathrm{~A})$ & 6163 & 4871 & 1967 & 75 \\
\hline $\mathrm{H}(27 \mathrm{~B})$ & 5356 & 4391 & 2937 & 75 \\
\hline $\mathrm{H}(28 \mathrm{~A})$ & 5917 & 2937 & 2921 & 80 \\
\hline $\mathrm{H}(28 \mathrm{~B})$ & 6709 & 3473 & 1955 & 80 \\
\hline $\mathrm{H}(29 \mathrm{~A})$ & 5718 & 3036 & 1228 & 80 \\
\hline $\mathrm{H}(29 B)$ & 6270 & 2158 & 2027 & 80 \\
\hline $\mathrm{H}(30 \mathrm{~A})$ & 4962 & 1192 & 2303 & 82 \\
\hline $\mathrm{H}(30 \mathrm{~B})$ & 4409 & 2014 & 1462 & 82 \\
\hline $\mathrm{H}(31 \mathrm{~A})$ & 3121 & 949 & 2529 & 82 \\
\hline $\mathrm{H}(31 \mathrm{~B})$ & 3286 & 1144 & 3327 & 82 \\
\hline $\mathrm{H}(32 \mathrm{~A})$ & 1315 & 1672 & 3433 & 76 \\
\hline $\mathrm{H}(32 \mathrm{~B})$ & 1268 & 1506 & 2588 & 76 \\
\hline $\mathrm{H}(33 \mathrm{~A})$ & 802 & 3096 & 1695 & 73 \\
\hline
\end{tabular}




$\begin{array}{lcccc}\mathrm{H}(33 \mathrm{~B}) & -103 & 2646 & 2608 & 73 \\ \mathrm{H}(35) & 9068 & 4757 & 628 & 80 \\ \mathrm{H}(36) & 8186 & 6128 & 474 & 108 \\ \mathrm{H}(37) & 6611 & 6688 & -119 & 113 \\ \mathrm{H}(38) & 5874 & 5874 & -592 & 102 \\ \mathrm{H}(39) & 6755 & 4461 & -450 & 83 \\ \mathrm{H}(40 \mathrm{~A}) & 8986 & 3662 & -417 & 161 \\ \mathrm{H}(40 \mathrm{~B}) & 8074 & 3123 & 448 & 161 \\ \mathrm{H}(40 \mathrm{C}) & 9141 & 3371 & 607 & 161 \\ \mathrm{H}(1 \mathrm{~A} 1) & 6824 & 6831 & 5560 & 37 \\ \mathrm{H}(1 \mathrm{~A} 2) & 8052 & 6910 & 4989 & 37 \\ \mathrm{H}(2 \mathrm{~A} 1) & 7576 & 8065 & 5501 & 42 \\ \mathrm{H}(2 \mathrm{~A} 2) & 6586 & 8491 & 4918 & 42 \\ & & & & \end{array}$




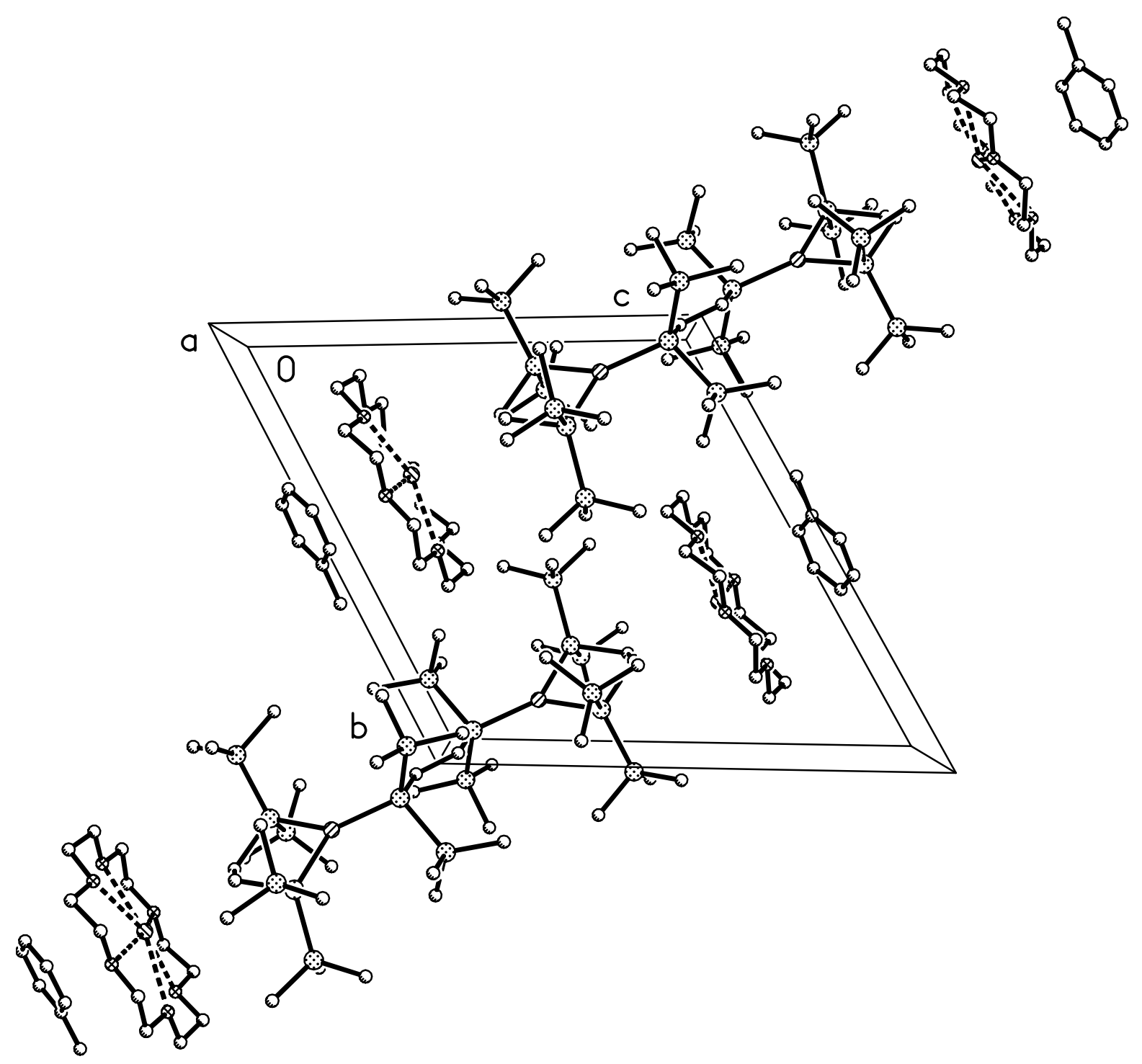

Figure S1. Packing diagram in 1. View down the $a$ axis 